\title{
Controlled release of encapsulated methylene blue in a multilayered textile coating
}

\author{
Adeline Martin ${ }^{1}$, Nicolas Tabary ${ }^{1}$, Laurent Leclercq ${ }^{2}$, Jatupol Junthip ${ }^{1}$, François Aubert- \\ Viard $^{3}$, Feng Chai ${ }^{3}$, Nicolas Blanchemain ${ }^{3}$ and Bernard Martel ${ }^{1}$ \\ 1 Unité Matériaux et Transformations (UMET) UMR CNRS 8207, Université de Lille1, France \\ 2 IBMM, UMR CNRS 5247, Université Montpellier 1, Montpellier, France \\ 3 INSERM U 1008, Controlled Drug Delivery Systems and Biomaterials, Lille, France
}

\begin{abstract}
SUMMARY
We studied the formation of multilayered coating incorporating a $\beta$-cyclodextrin polyelectrolyte onto a pretreated polyethylene terephthalate (PET) textile in order to obtain reservoir and sustained release properties towards bioactive molecules. This paper describes the alternate deposition by dip-coating onto the textile of chitosan (CHT) and a $\beta$-cyclodextrin polyelectrolyte (polyCTR$\beta \mathrm{CD}$ ) according to the layer-by-layer (LbL) principle. Textiles covered with up to 12 layers were characterized by gravimetry, infrared, zetametry. The building of the multilayer system was then achieved including methylene blue (MB) as bioactive model compound, complexed with polyCTR- $\beta \mathrm{CD}$, and a release study of $\mathrm{BM}$ was investigated in batch. The results showed that the release profile of BM could be controlled by the number of layers in the system.
\end{abstract}

\section{INTRODUCTION}

In the last two decades, surface treatments of biomaterials have been investigated aiming to modify their physicochemical properties to control cell proliferation or adhesion, or to create drug delivery system (GarcíaGonzález et al. 2011). In this case, the main challenges are firstly to chemically modify such inert materials with soft methods in order to keep their original properties and secondly to adsorb a sufficient therapeutic dose of the drug that should then be released covering the critical healing period.

Cyclodextrins (CDs) and their polymers have proved to be the appropriate candidates for this purpose (Uekama et al. 1998). Due to the hydrophobic character of their cavities, they are known to form reversible inclusion complexes with many hydrophobic bio-active molecules and promote their solubility. So, CDs are widely used for their encapsulation properties as drug carriers ( $\mathrm{Li}$ et al. 2004, Sun et al. 2012).

The aim of the present study is to build an innovative system of a multilayer assembly on a pretreated PET using the LbL process, using a polymer of $\beta$ cyclodextrin $(\beta \mathrm{CD})$ as a polyanion with an active molecule encapsulated in the cavities, and chitosan as a polycation, a biosourced biocompatible polymer. The LbL technique, developed by Decher over the past several years (Decher et al. 1992), is fast, easy to use, with a soft and biocompatible process (mainly in water) and is adjustable to several substrates.

This system was physically, chemically and biologically tested as drug delivery system.

\section{EXPERIMENTAL PART}

Non-woven PET, $\left(65 \mathrm{~g} / \mathrm{m}^{2}\right)$ was pre-treated by a high cross-linked copolymer of $\beta \mathrm{CD}$ and citric acid bearing anionic groups onto the surface (Martin et al. 2013). The multilayer assembly was then built by the dip-coating method (Decher et al. 1992). Pre-treated samples were alternatively dipped into a chitosan polycation solution, and a polyanion cyclodextrin polymer (poly- $\beta$ CD, Fig. 1). Up to 12 layers were deposited. The same process was used, incorporating methylene blue as drug model (MB) in the system. The building of the multilayer assembly was monitored by Optical Waveguide Lightmode Spectroscopy (OWLS) and characterized by zetametry, microscopy (SEM) and physicochemical methods (FTIR, TGA, gravimetry). Biocompatibility was proved by culture in vitro of L132 cells. Release of MB was followed in distilled water and PBS solution by UV-visible spectroscopy.

\section{RESULTS AND DISCUSSION}

OWLS, zetametry and gravimetry confirmed that chitosan and poly- $\beta C D$ were alternatively deposited on the surface. MB slightly disturbed the multilayer building due to its cationic character (Fig. 2).

SEM showed a significant partial filling of the gaps due to the fibers coating. The release study of MB showed a relation between the number of layers and the amount of released MB in the supernatant (Fig. 3). The layer by layer assembly allowed the sustained release of MB as no burst effect was observed. On the contrary, MB release occurred with a regular rate over 3 months.

Proliferation tests with L132 cells on samples coated with up to 12 layers proved their biocompatibility. 


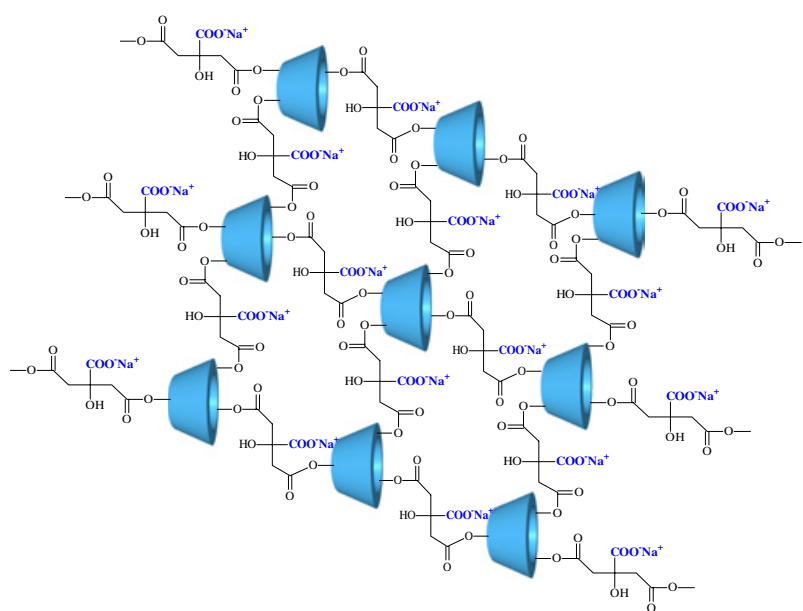

Figure 1. Structure of polyCTR- $\beta \mathrm{CD}$.

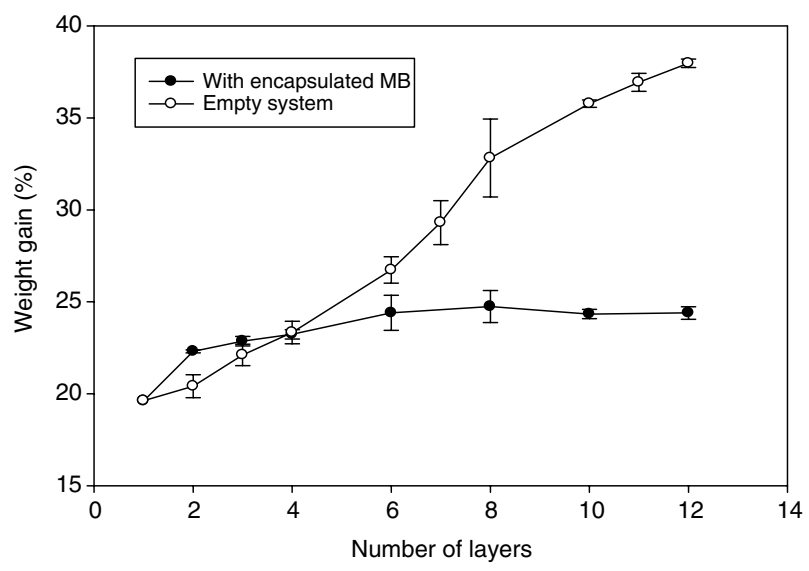

Figure 2. Comparative evolution of the cumulative mass on assemblies with or without MB.

\section{CONCLUSION}

We realized the controlled deposition of chitosan and cyclodextrin polyelectrolytes onto a textile support. The

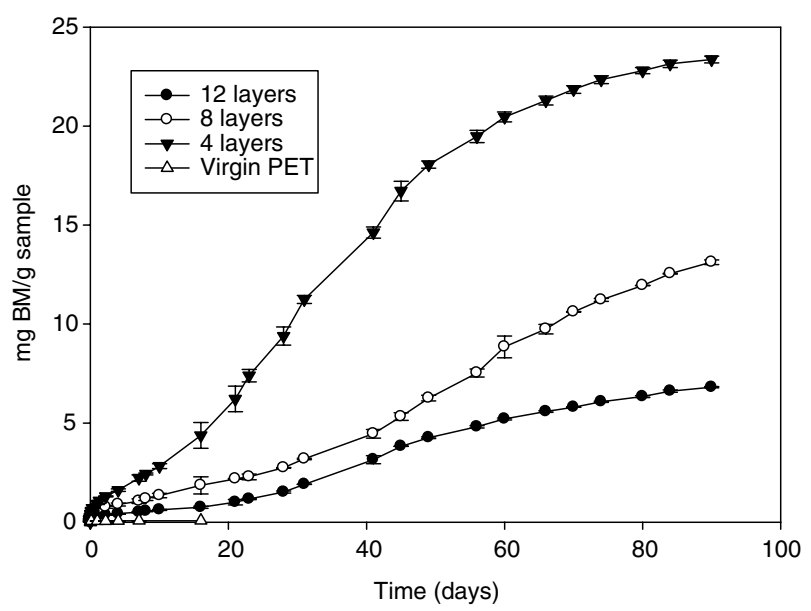

Figure 3. Release study of MB in multilayers assemblies in water at $37^{\circ} \mathrm{C}$ under stirring.

$\mathrm{L}-b$ - $\mathrm{L}$ system allowed the control of the dose and the sustained release of MB. The biocompatibility of the system will allow us to investigate clinical applications, such as wound-healings, where antibacterial and healing properties of both used polyelectrolytes could offer a relevant added-value.

\section{References}

Decher, G., et al. (1992). Thin Solid Films 210-211, Part 2(0): 831-835.

García-González, C., et al. (2011). Carbohydr. Polym. 86(4): 1425-1438

Li, J., et al. (2004). Int. J. Pharm. 278(2): 329-342.

Martin, A., et al. (2013). Carbohydr. Polym. 93: 718-730

Sun, T., et al. (2012). Langmuir, 28(23): 8625-8636.

Uekama, K., et al. (1998). Chem. Rev. (Washington, DC, U. S.) 98(5): 2045-2076. 